\title{
Implementation of electronic patient clinical records in ambulances in the UK: a national survey.
}

WILLIAMS, V., LAFLAMME-WILLIAMS, Y., MCNEE, K., MORGAN, H., MORRISON, Z., POTTS, H.W.W., SHAW, D., SIRIWARDENA, N., SNOOK, H., SPAIGHT, R. and PORTER, A. 


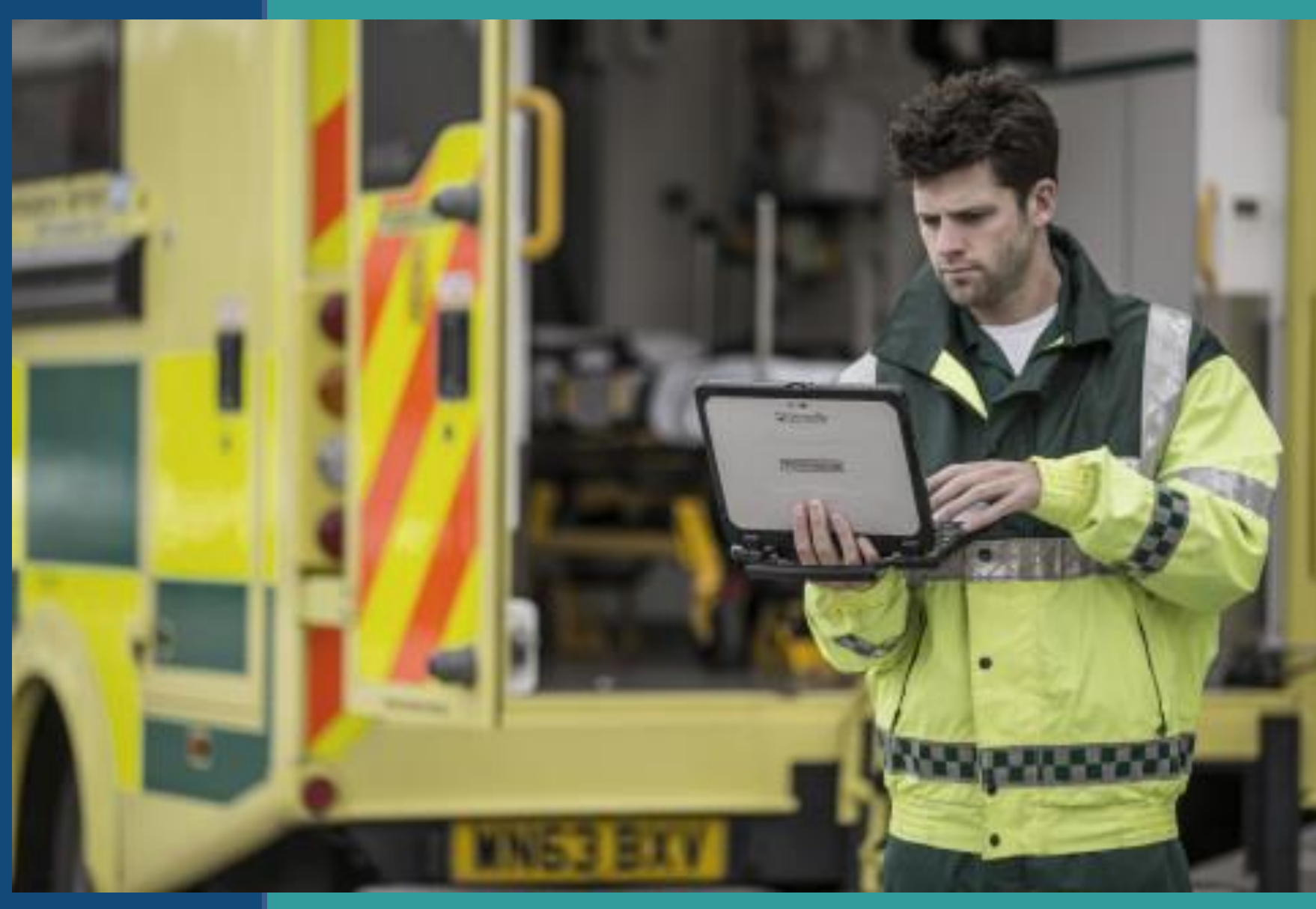

Background

The roll-out of electronic Patient Clinical Records (ePCR) across UK ambulance services has been an important aspect of modernisation. Electronic Records in Ambulances (ERA) is a two-year study which aims to describe the opportunities and challenges of implementing ePCR and associated technology in emergency ambulances.

Our study includes a baseline survey of progress implementing ePCR in all UK ambulance services providing a snapshot of current usage.

\section{Methods}

We carried out semi-structured telephone interviews with information managers in each ambulance service in the UK. We asked them about the systems in use, the process and current stage of implementation and explored the perceived value of using ePCR. If services did not use ePCR we asked about plans for future introduction.

The interviews were transcribed and thematically analysed, by three members of the research team.

\section{Implementation of} electronic patient clinical records in ambulances in the UR: a national survey

\section{Victoria Williams Swansea University}

Yvette LaFlamme Williams Welsh Ambulance Service

Katie McNee South West Ambulance Service

Robert Harris-Mayes SUCCESS service user group

Heather Morgan Aberdeen University

Zoe Morrison Aberdeen University

Henry Potts University College London

Debbie Shaw East Midlands Ambulance Service

Niro Siriwardena Lincoln University and East Midlands Ambulance Service Helen Snooks Swansea University

Rob Spaight East Midlands Ambulance Service

Alison Porter Swansea University

\section{Results}

We completed interviews with 22 managers from all 13 services.

Implementation varied across the UK.

Seven services were using electronic records. Four services had adopted electronic records but, at the time of interview, had reverted to paper with the intention of implementing a new ePCR. Two services still used paper but hoped to move to ePCR in the future.

Those who had fully implemented ePCR reported mixed success in terms of staff compliance, and in realising the potential benefits offered by ePCR to link with primary and secondary care.

Potential improvements to patient care were discussed, but tended to be associated with future planned developments.

\section{Implications}

Implementation of ePCR has proved challenging with wide variation in use between ambulance services. Progress has been erratic, rather than linear, demonstrated by difficulties that services experienced putting ePCR into practice.

Reported benefits of ePCR were largely associated with improved data management for audit and record keeping.

Levels of interconnectivity between ambulances and other health care providers were similarly varied but this was something that all wanted to improve in the future.

There is potential for ambulance services in the earlier stages of implementation to learn from the experiences of others.

\section{ERA}

Electronic

Records in

Ambulances

This project was funded by the National Institute for Health Research HS\&DR programme (project number 14/47/22). The views and opinions those of the HS\&DR programme, NIHR, NHS or the Department of Health

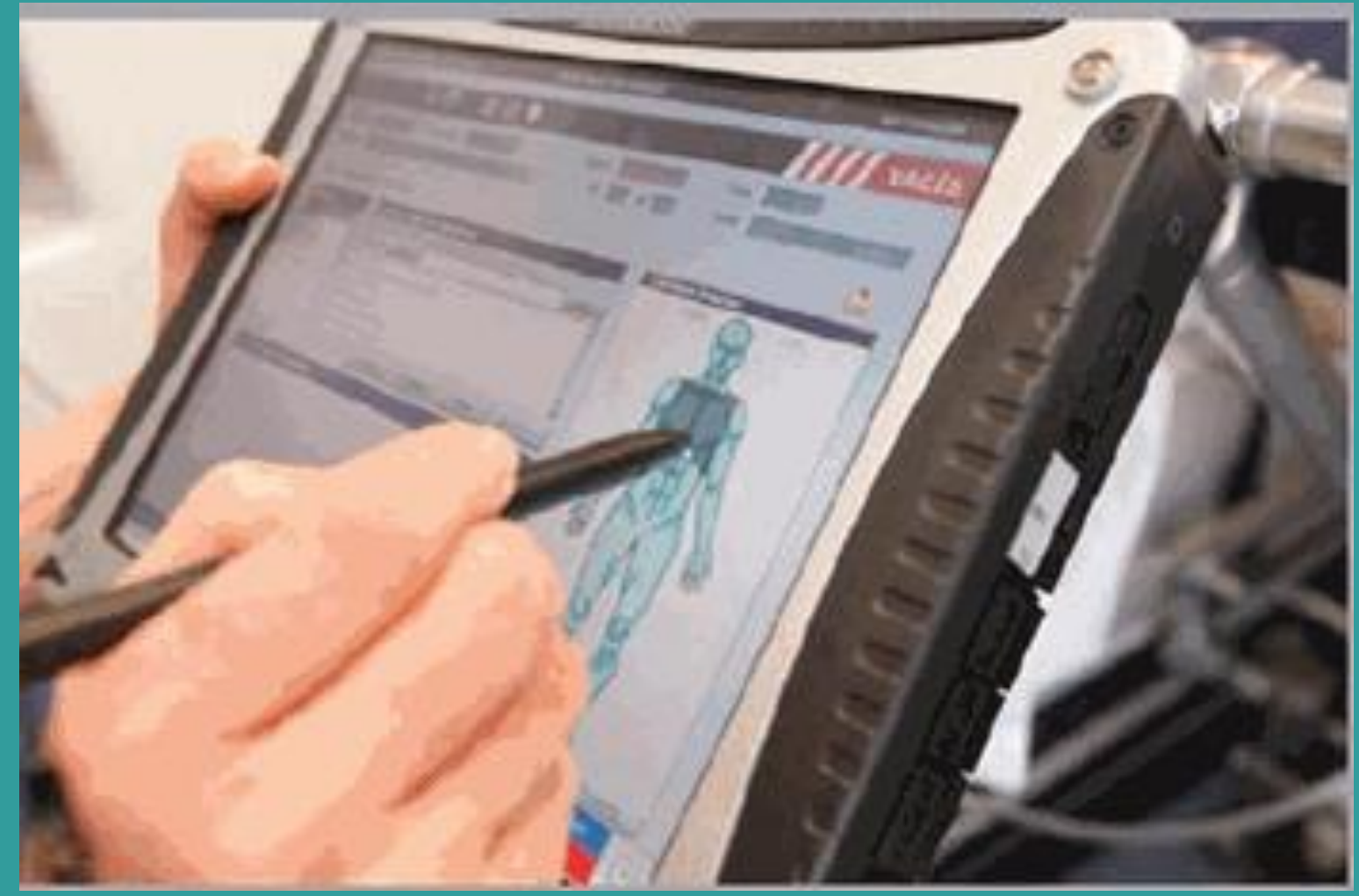

